\title{
Compact differences of weighted composition operators
}

\author{
Bin Liu ${ }^{1}$ (D) . Jouni Rättyä ${ }^{1}$
}

Received: 6 March 2020 / Accepted: 28 November 2020 / Published online: 11 December 2020

(c) The Author(s) 2020

\begin{abstract}
Compact differences of two weighted composition operators acting from the weighted Bergman space $A_{\omega}^{p}$ to another weighted Bergman space $A_{v}^{q}$, where $0<p \leq q<\infty$ and $\omega, v$ belong to the class $\mathcal{D}$ of radial weights satisfying two-sided doubling conditions, are characterized. On the way to the proof a new description of $q$-Carleson measures for $A_{\omega}^{p}$, with $\omega \in \mathcal{D}$, in terms of pseudohyperbolic discs is established. This last-mentioned result generalizes the well-known characterization of $q$-Carleson measures for the classical weighted Bergman space $A_{\alpha}^{p}$ with $-1<\alpha<\infty$ to the setting of doubling weights.
\end{abstract}

Keywords Bergman space · Doubling weight - Weighted composition operator · Compactness

Mathematics Subject Classification $47 \mathrm{~B} 33 \cdot 30 \mathrm{H} 20$

\section{Introduction and main results}

Let $\mathcal{H}(\mathbb{D})$ denote the space of analytic functions in the unit disc $\mathbb{D}=\{z \in \mathbb{C}:|z|<1\}$. For a nonnegative function $\omega \in L^{1}([0,1))$, the extension to $\mathbb{D}$, defined by $\omega(z)=\omega(|z|)$ for all $z \in \mathbb{D}$, is called a radial weight. For $0<p<\infty$ and a radial weight $\omega$, the weighted Bergman space $A_{\omega}^{p}$ consists of $f \in \mathcal{H}(\mathbb{D})$ such that

$$
\|f\|_{A_{\omega}^{p}}^{p}=\int_{\mathbb{D}}|f(z)|^{p} \omega(z) d A(z)<\infty,
$$

where $d A(z)=\frac{d x d y}{\pi}$ is the normalized Lebesgue area measure on $\mathbb{D}$. As usual, $A_{\alpha}^{p}$ stands for the classical weighted Bergman space induced by the standard radial weight $\omega(z)=\left(1-|z|^{2}\right)^{\alpha}$, where $-1<\alpha<\infty$.

The first author is supported by China Scholarship Council (No.201706330108).

Bin Liu

binl@uef.fi

Jouni Rättyä

jouni.rattya@uef.fi

1 Department of Physics and Mathematics, University of Eastern Finland, P.O.Box 111, 80101 Joensuu, Finland 
For a radial weight $\omega$, write $\widehat{\omega}(z)=\int_{|z|}^{1} \omega(s) d s$ for all $z \in \mathbb{D}$. In this paper we always assume $\widehat{\omega}(z)>0$, for otherwise $A_{\omega}^{p}=\mathcal{H}(\mathbb{D})$ for each $0<p<\infty$. A weight $\omega$ belongs to the class $\widehat{\mathcal{D}}$ if there exists a constant $C=C(\omega) \geq 1$ such that $\widehat{\omega}(r) \leq C \hat{\omega}\left(\frac{1+r}{2}\right)$ for all $0 \leq r<1$. Moreover, if there exist $K=K(\omega)>1$ and $C=C(\omega)>1$ such that $\widehat{\omega}(r) \geq C \hat{\omega}\left(1-\frac{1-r}{K}\right)$ for all $0 \leq r<1$, then we write $\omega \in \check{\mathcal{D}}$. In other words, $\omega \in \check{\mathcal{D}}$ if there exists $K=K(\omega)>1$ and $C^{\prime}=C^{\prime}(\omega)>0$ such that

$$
\widehat{\omega}(r) \leq C^{\prime} \int_{r}^{1-\frac{1-r}{K}} \omega(t) d t, \quad 0 \leq r<1 .
$$

The intersection $\widehat{\mathcal{D}} \cap \breve{\mathcal{D}}$ is denoted by $\mathcal{D}$, and this is the class of weights that we mainly work with.

Each analytic self-map $\varphi$ of $\mathbb{D}$ induces the composition operator $C_{\varphi}$ on $\mathcal{H}(\mathbb{D})$ defined by $C_{\varphi} f=f \circ \varphi$. The weighted composition operator induced by $u \in \mathcal{H}(\mathbb{D})$ and $\varphi$ is $u C_{\varphi}$ and sends $f \in \mathcal{H}(\mathbb{D})$ to $u \cdot f \circ \varphi \in \mathcal{H}(\mathbb{D})$. These operators have been extensively studied in a variety of function spaces (see for example [4-8, 22, 23, 25, 26]).

If now $\psi$ is another analytic self-map of $\mathbb{D}$, the pair $(\varphi, \psi)$ induces the operator $C_{\varphi}-C_{\psi}$. One of the most important problem considering these operators is to characterize compact differences in Hardy spaces. Shapiro and Sundberg [24] studied this problem in 1990. Very recently, Choe, Choi, Koo and Yang [3] have solved this problem. For more about difference operators, see [2, 8, 10, 11, 20]. Moorhouse [11, 12] obtain some important results on this operator in weighted Bergman spaces. He showed [11], among other things, that $C_{\varphi}-C_{\psi}$ is compact on $A_{\alpha}^{2}$ if and only if

$$
\lim _{|z| \rightarrow 1^{-}}\left|\delta_{1}(z)\right|\left(\frac{1-|z|^{2}}{1-|\varphi(z)|^{2}}+\frac{1-|z|^{2}}{1-|\psi(z)|^{2}}\right)=0
$$

where

$$
\delta_{1}(z)=\frac{\varphi(z)-\psi(z)}{1-\overline{\varphi(z)} \psi(z)}, \quad z \in \mathbb{D} .
$$

Saukko $[20,21]$ generalized this result by showing that if either $1<p \leq q$, or $p>q \geq 1$, then $C_{\varphi}-C_{\psi}: A_{\alpha}^{p} \rightarrow A_{\beta}^{q}$ is compact if and only if the operators $\delta_{1} C_{\varphi}$ and $\delta_{1} C_{\psi}$ are both compact from $A_{\alpha}^{p}$ to $L_{\beta}^{q}$. Very recently, Acharyya and Wu [1] characterized the compact differences of two weighted composition operators $u C_{\varphi}-v C_{\psi}$ between different weighted Bergman spaces $A_{\alpha}^{p}$ and $A_{\beta}^{q}$, where $0<p \leq q<\infty$ and $-1<\alpha, \beta<\infty$. Their result states that, if $\frac{\alpha+2}{p} \leq \frac{\beta+2}{q}$ and $u, v \in \mathcal{H}(\mathbb{D})$ satisfy

$$
\sup _{z \in \mathbb{D}}(|u(z)|+|v(z)|)\left(1-|z|^{2}\right)^{\frac{\beta+2}{q}-\frac{\alpha+2}{p}}<\infty
$$

then $u C_{\varphi}-v C_{\psi}: A_{\alpha}^{p} \rightarrow A_{\beta}^{q}$ is compact if and only if

$$
\lim _{|z| \rightarrow 1^{-}}\left|\delta_{1}(z)\right|\left(|u(z)| \frac{\left(1-|z|^{2}\right)^{\frac{2+\beta}{q}}}{\left(1-|\varphi(z)|^{2}\right)^{\frac{2+\alpha}{p}}}+|v(z)| \frac{\left(1-|z|^{2}\right)^{\frac{2+\beta}{q}}}{\left(1-|\psi(z)|^{2}\right)^{\frac{2+\alpha}{p}}}\right)=0
$$

and 


$$
\lim _{|z| \rightarrow 1^{-}}\left(1-\left|\delta_{1}(z)\right|^{2}\right)^{\frac{2+\alpha}{p}}|u(z)-v(z)|\left(\frac{\left(1-|z|^{2}\right)^{\frac{2+\beta}{q}}}{\left(1-|\varphi(z)|^{2}\right)^{\frac{2+\alpha}{p}}}+\frac{\left(1-|z|^{2}\right)^{\frac{2+\beta}{q}}}{\left(1-|\psi(z)|^{2}\right)^{\frac{2+\alpha}{p}}}\right)=0 .
$$

In this paper we characterize compact differences of two weighted composition operators from the weighted Bergman space $A_{\omega}^{p}$ to another weighted Bergman space $A_{v}^{q}$ with $0<p \leq q<\infty$ and $\omega, \nu \in \mathcal{D}$. To state the result, write

$$
\delta_{2}(z)=\frac{\psi(z)-\varphi(z)}{1-\overline{\psi(z)} \varphi(z)}, \quad z \in \mathbb{D},
$$

and observe that $\left|\delta_{1}\right|=\left|\delta_{2}\right|$ on $\mathbb{D}$. Our main result reads as follows.

Theorem 1 Let $\omega, v \in \mathcal{D}$ and $0<p \leq q<\infty$ such that $\widehat{v}(z)(1-|z|) \lesssim(\widehat{\omega}(z)(1-|z|))^{\frac{q}{p}}$ for all $z \in \mathbb{D}$. Further, let $u, v \in \mathcal{H}(\mathbb{D})$ and $\varphi$ and $\psi$ analytic self-maps of $\mathbb{D}$ such that

$$
\sup _{z \in \mathbb{D}}(|u(z)|+|v(z)|) \frac{(\widehat{v}(z)(1-|z|))^{\frac{1}{q}}}{(\widehat{\omega}(z)(1-|z|))^{\frac{1}{p}}}<\infty .
$$

Then there exists $\gamma=\gamma(\omega, p)>0$ with the following property: $u C_{\varphi}-v C_{\psi}: A_{\omega}^{p} \rightarrow A_{v}^{q}$ is compact if and only if

$$
\lim _{|z| \rightarrow 1^{-}}\left|\delta_{1}(z)\right|\left(|u(z)| \frac{(\widehat{v}(z)(1-|z|))^{\frac{1}{q}}}{(\widehat{\omega}(\varphi(z))(1-|\varphi(z)|))^{\frac{1}{p}}}+|v(z)| \frac{(\widehat{v}(z)(1-|z|))^{\frac{1}{q}}}{(\widehat{\omega}(\psi(z))(1-|\psi(z)|))^{\frac{1}{p}}}\right)=0
$$

and

$$
\begin{aligned}
& \lim _{|z| \rightarrow 1^{-}}\left(\frac{\left|1-\overline{\varphi(z)} \delta_{1}(z)\right|^{\gamma}}{(\widehat{\omega}(\varphi(z))(1-|\varphi(z)|))^{\frac{1}{p}}}+\frac{\left|1-\overline{\psi(z)} \delta_{2}(z)\right|^{\gamma}}{(\widehat{\omega}(\psi(z))(1-|\psi(z)|))^{\frac{1}{p}}}\right) \\
& \cdot|u(z)-v(z)|(\widehat{v}(z)(1-|z|))^{\frac{1}{q}}=0 .
\end{aligned}
$$

If $\omega(z)=(1-|z|)^{\alpha}$ and $v(z)=(1-|z|)^{\beta}$ for $-1<\alpha, \beta<\infty$, then $\widehat{\omega}(z) \asymp(1-|z|)^{\alpha+1}$ and $\widehat{v}(z) \asymp(1-|z|)^{\beta+1}$ for all $z \in \mathbb{D}$. Therefore (5) reduces to (2). Moreover, the Proof of Theorem 1 shows that the only requirement for $\gamma=\gamma(\omega, p)>0$ appearing in the statement is that

$$
\int_{\mathbb{D}} \frac{\omega(z)}{|1-\bar{a} z|^{\gamma p}} d A(z) \leq C \frac{\widehat{\omega}(a)}{(1-|a|)^{\gamma p-1}}, \quad a \in \mathbb{D},
$$

for some constant $C=C(\omega, p, \gamma)>0$. If $\omega(z)=(1-|z|)^{\alpha}$, any $\gamma>\frac{\alpha+2}{p}$ is acceptable, and the choice $\gamma=2 \frac{\alpha+2}{p}$ converts (6) to (3), as a simple computation shows. Therefore Theorem 1 indeed generalizes [1, Theorem 1] for weights in $\mathcal{D}$.

We need two specific tools for the Proof of Theorem 1. The first one concerns continuous embeddings $A_{\omega}^{p} \subset L_{\mu}^{q}$. Recall that a positive Borel measure $\mu$ on $\mathbb{D}$ is a $q$-Carleson measure for $A_{\omega}^{p}$ if the identity operator $I_{d}: A_{\omega}^{p} \rightarrow L_{\mu}^{q}$ is bounded. A complete characterization of such measures in the case $\omega \in \widehat{\mathcal{D}}$ can be found in [15], see also [13, 17]. In particular, it is known that if $q \geq p$ and $\omega \in \widehat{\mathcal{D}}$, then $\mu$ is a $q$-Carleson measure for $A_{\omega}^{p}$ if and only if 


$$
\sup _{a \in \mathbb{D}} \frac{\mu(S(a))}{\omega(S(a))^{\frac{q}{p}}}<\infty .
$$

Here and from now on $S(a)=\{z: 1-|a|<|z|<1,|\arg z-\arg a|<(1-|a|) / 2\}$ is the Carleson square induced by the point $a \in \mathbb{D} \backslash\{0\}, S(0)=\mathbb{D}$ and $\omega(E)=\int_{E} \omega d A$ for each measurable set $E \subset \mathbb{D}$. We will need a variant of this result and its "compact" counterpart for $\omega \in \mathcal{D}$ where the Carleson squares are replaced by pseudohyperbolic discs. To this end, denote $\varphi_{a}(z)=\frac{a-z}{1-\bar{a} z}$ for $a, z \in \mathbb{D}$. The pseudohyperbolic distance between two points $a$ and $b$ in $\mathbb{D}$ is $\rho(a, b)=\left|\varphi_{a}(b)\right|$. For $a \in \mathbb{D}$ and $0<r<1$, the pseudohyperbolic disc of center $a$ and of radius $r$ is $\Delta(a, r)=\{z \in \mathbb{D}: \rho(a, z)<r\}$. It is well known that $\Delta(a, r)$ is an Euclidean disk centered at $\left(1-r^{2}\right) a /\left(1-r^{2}|a|^{2}\right)$ and of radius $\left(1-|a|^{2}\right) r /\left(1-r^{2}|a|^{2}\right)$.

Theorem 2 Let $0<p \leq q<\infty, \omega \in \mathcal{D}$ and $\mu$ a positive Borel measure on $\mathbb{D}$. Then there exists $r=r(\omega) \in(0,1)$ such that the following statements hold:

(i) $\mu$ is a q-Carleson measure for $A_{\omega}^{p}$ if and only if

$$
\sup _{a \in \mathbb{D}} \frac{\mu(\Delta(a, r))}{(\omega(\Delta(a, r)))^{\frac{q}{p}}}<\infty .
$$

Moreover, if $\mu$ is a $q$-Carleson measure for $A_{\omega}^{p}$, then the identity operator satisfies

$$
\left\|I_{d}\right\|_{A_{\omega}^{p} \rightarrow L_{\mu}^{q}}^{q} \asymp \sup _{a \in \mathbb{D}} \frac{\mu(\Delta(a, r))}{(\omega(\Delta(a, r)))^{\frac{q}{p}}} .
$$

(ii) The identity operator $I_{d}: A_{\omega}^{p} \rightarrow L_{\mu}^{q}$ is compact if and only if

$$
\lim _{|a| \rightarrow 1^{-}} \frac{\mu(\Delta(a, r))}{(\omega(\Delta(a, r)))^{\frac{q}{p}}}=0 .
$$

Another result needed is a lemma that allows us to estimate the distance between images of two points, say $z$ and $a$, under $f$ sufficiently accurately whenever $z$ is close to $a$ in the sense that $z \in \Delta(a, r)$, and $f \in A_{\omega}^{p}$ with $\omega \in \mathcal{D}$. For the statement, denote $\widetilde{\omega}(z)=\widehat{\omega}(z) /(1-|z|)$ for all $z \in \mathbb{D}$.

Lemma 1 Let $\omega \in \mathcal{D}, 0<p \leq q<\infty$ and $0<r<R<1$. Then there exists a constant $C=C(\omega, p, q, r, R)>0$ such that

$$
|f(z)-f(a)|^{q} \leq C \frac{\rho(z, a)^{q}}{(\widehat{\omega}(a)(1-|a|))^{\frac{q}{p}}} \int_{\Delta(a, R)}|f(\zeta)|^{p} \widetilde{\omega}(\zeta) d A(\zeta), \quad a \in \mathbb{D}, \quad z \in \Delta(a, r),
$$

for all $f \in A_{\omega}^{p}$ with $\|f\|_{A_{\omega}^{p}} \leq 1$.

This lemma plays an important role in the Proof of Theorem 1 when we show that (5) and (6) are sufficient conditions for the compactness. By [18, Proposition 5] we know that 


$$
\|f\|_{A_{\widetilde{\omega}}^{p}} \asymp\|f\|_{A_{\omega}^{p}}, \quad f \in \mathcal{H}(\mathbb{D}),
$$

provided $\omega \in \mathcal{D}$. This explains the appearance of the weight $\widetilde{\omega}$ on the right hand side of (9). It is worth observing that, despite of (10), the strictly positive weight $\widetilde{\omega}$ cannot be replaced by $\omega$ in the statement because $\omega \in \mathcal{D}$ may vanish in pseudohyperbolic discs of fixed radius that tend to the boundary.

The rest of the paper contains the proofs of the results stated above. We first prove Lemma 1 in the next section. The proof of the result on Carleson measures, Theorem 2, is given in Sect. 3, and finally, Theorem 1 is proved in Sect. 4.

To this end, couple of words about the notation used in the sequel. The letter $C=C(\cdot)$ will denote an absolute constant whose value depends on the parameters indicated in the parenthesis, and may change from one occurrence to another. We will use the notation $a \lesssim b$ if there exists a constant $C=C(\cdot)>0$ such that $a \leq C b$, and $a \gtrsim b$ is understood in an analogous manner. In particular, if $a \lesssim b$ and $a \gtrsim b$, then we write $a \asymp b$ and say that $a$ and $b$ are comparable.

\section{Proof of Lemma 1}

It is known that if $\omega \in \mathcal{D}$, then there exist constants $0<\alpha=\alpha(\omega) \leq \beta(\omega)<\infty$ and $C=C(\omega) \geq 1$ such that

$$
\frac{1}{C}\left(\frac{1-r}{1-t}\right)^{\alpha} \leq \frac{\widehat{\omega}(r)}{\widehat{\omega}(t)} \leq C\left(\frac{1-r}{1-t}\right)^{\beta}, \quad 0 \leq r \leq t<1 .
$$

In fact, this pair of inequalities characterizes the class $\mathcal{D}$ because the right hand inequality is satisfied if and only if $\omega \in \widehat{\mathcal{D}}$ by [17, Lemma 2.1] while the left hand inequality describes the class $\check{\mathcal{D}}$ in an analogous way, see [14, (2.27)]. The chain of inequalities (11) will be frequently used in the sequel.

To prove the lemma, let $a \in \mathbb{D}, 0<r<1$ and $z \in \Delta(a, r)$. Then

$$
\begin{aligned}
|f(z)-f(a)|^{p} & =\left|f\left(\varphi_{a}\left(\varphi_{a}(z)\right)\right)-f\left(\varphi_{a}(0)\right)\right|^{p}=\left|\int_{0}^{\varphi_{a}(z)}\left(f \circ \varphi_{a}\right)^{\prime}(\xi) d \xi\right|^{p} \\
& \leq \max _{\xi \in D\left(0,\left|\varphi_{a}(z)\right|\right)}\left|\left(f \circ \varphi_{a}\right)^{\prime}(\xi)\right|^{p}\left|\varphi_{a}(z)\right|^{p} \leq \max _{\xi \in \overline{D(0, r)}}\left|\left(f \circ \varphi_{a}\right)^{\prime}(\xi)\right|^{p}\left|\varphi_{a}(z)\right|^{p} .
\end{aligned}
$$

Let $R \in(r, 1)$ and set $R^{\prime}=\frac{r+R}{2}$. Further, let $0<s<1$. Then the Cauchy integral formula for the derivative and the subharmonicity of $|f|^{p}$ yield

$$
\begin{aligned}
\left|\left(f \circ \varphi_{a}\right)^{\prime}(\xi)\right|^{p} & =\left|\frac{1}{2 \pi} \int_{|w|=R^{\prime}} \frac{\left(f \circ \varphi_{a}\right)(w)}{(w-\xi)^{2}} d w\right|^{p} \leq\left(\frac{2}{R-r}\right)^{2 p}\left(R^{\prime}\right)^{p} \max _{|w|=R^{\prime}}\left|f\left(\varphi_{a}(w)\right)\right|^{p} \\
& \lesssim \frac{4}{\pi s^{2}} \max _{|w|=R^{\prime}} \frac{1}{\left(1-\left|\varphi_{a}(w)\right|\right)^{2}} \int_{\Delta\left(\varphi_{a}(w), s\right)}|f(\zeta)|^{p} d A(\zeta) \\
& \lesssim \max _{|w|=R^{\prime}} \frac{1}{(1-|a|)^{2}} \int_{\Delta\left(\varphi_{a}(w), s\right)}|f(\zeta)|^{p} d A(\zeta), \quad \xi \in \overline{D(0, r)} .
\end{aligned}
$$

Fix now $s=s(r, R) \in(0,1)$ sufficiently small such that $\Delta\left(\varphi_{a}(w), s\right) \subset \Delta(a, R)$ for all $w$ such that $|w|=R^{\prime}$. Further, an application of the right hand inequality in (11) shows that $\widehat{\omega}(\zeta) \asymp \widehat{\omega}(a)$ for all $\zeta \in \Delta(a, R)$. Therefore, by combining (12) and (13) we deduce 


$$
\begin{aligned}
|f(z)-f(a)|^{p} & \lesssim \frac{\left|\varphi_{a}(z)\right|^{p}}{(1-|a|)^{2}} \int_{\Delta(a, R)}|f(\zeta)|^{p} d A(\zeta) \\
& \lesssim \frac{\left|\varphi_{a}(z)\right|^{p}}{\widehat{\omega}(a)(1-|a|)} \int_{\Delta(a, R)}|f(\zeta)|^{p} \widetilde{\omega}(\zeta) d A(\zeta), \quad a \in \mathbb{D}, \quad z \in \Delta(a, r) .
\end{aligned}
$$

This proves the case $p=q$ because $\left|\varphi_{a}(z)\right|=\rho(z, a)$ for all $a, z \in \mathbb{D}$. This part of the proof is valid for all $f \in \mathcal{H}(\mathbb{D})$ if $\omega \in \widehat{\mathcal{D}}$.

Let now $q>p$, and observe that trivially $|f(z)-f(a)|^{q}=\left(|f(z)-f(a)|^{p}\right)^{\frac{q}{p}}$. An application of the case $q=p$ implies

$$
\begin{aligned}
|f(z)-f(a)|^{q} & \lesssim \frac{\rho(z, a)^{q}}{(\widehat{\omega}(a)(1-|a|))^{\frac{q}{p}}}\left(\int_{\Delta(a, R)}|f(\zeta)|^{p} \widetilde{\omega}(\zeta) d A(\zeta)\right)^{\frac{q}{p}} \\
& \leq \frac{\rho(z, a)^{q}|| f \|_{A_{\widetilde{\omega}}^{q}}^{q-p}}{(\widehat{\omega}(a)(1-|a|))^{\frac{q}{p}}} \int_{\Delta(a, R)}|f(\zeta)|^{p} \widetilde{\omega}(\zeta) d A(\zeta) .
\end{aligned}
$$

But (10) guarantees $\|f\|_{A_{\tilde{\omega}}^{p}} \asymp\|f\|_{A_{\omega}^{p}} \leq 1$, and thus the assertion in the case $q>p$ follows from the above estimate.

\section{Proof of Theorem 2}

To prove (i), assume first (7) and let $0<r<1$. The fact that $|f|^{p}$ is subharmonic in $\mathbb{D}$ together with Minkowski's inequality in continuous form (Fubini's theorem in the case $q=p$ ) and (7) imply

$$
\begin{aligned}
\|f\|_{L_{\mu}^{q}}^{q} & \lesssim \int_{\mathbb{D}}\left(\int_{\Delta(z, r)} \frac{|f(\zeta)|^{p}}{(1-|\zeta|)^{2}} d A(\zeta)\right)^{\frac{q}{p}} d \mu(z) \\
& \leq\left(\int_{\mathbb{D}}|f(\zeta)|^{p} \frac{\mu(\Delta(\zeta, r))^{\frac{p}{q}}(1-|\zeta|)^{2}}{d A(\zeta))^{\frac{q}{p}}}\right. \\
& \lesssim\left(\int_{\mathbb{D}}|f(\zeta)|^{p} \frac{\omega(\Delta(\zeta, r))}{(1-|\zeta|)^{2}} d A(\zeta)\right)^{\frac{q}{p}}, \quad f \in \mathcal{H}(\mathbb{D}) .
\end{aligned}
$$

Since $\omega \in \mathcal{D}$ by the hypothesis, we may apply the right hand inequality in (11) to deduce

$$
\omega(\Delta(\zeta, r)) \lesssim \widehat{\omega}(\zeta)(1-|\zeta|), \quad \zeta \in \mathbb{D} .
$$

It follows that $\|f\|_{L_{\mu}^{q}} \lesssim\|f\|_{A_{\tilde{\omega}}^{p}}$, and hence $\|f\|_{L_{\mu}^{q}} \lesssim\|f\|_{A_{\omega}^{p}}$ for all $f \in \mathcal{H}(\mathbb{D})$ by (10). Thus $\mu$ is a $q$-Carleson measure $A_{\omega}^{p}$.

Conversely, assume that $\mu$ is a $q$-Carleson measure $A_{\omega}^{p}$. For each $a \in \mathbb{D}$, consider the function

$$
f_{a}(z)=\left(\frac{1-|a|^{2}}{1-\bar{a} z}\right)^{\gamma} \frac{1}{(\widehat{\omega}(a)(1-|a|))^{\frac{1}{p}}}=\frac{\left(1-\bar{a} \varphi_{a}(z)\right)^{\gamma}}{(\widehat{\omega}(a)(1-|a|))^{\frac{1}{p}}}, \quad z \in \mathbb{D},
$$


induced by $\omega$ and $0<\gamma, p<\infty$. Then [17, Lemma 2.1] implies that for all $\gamma=\gamma(\omega, p)>0$ sufficiently large we have $\left\|f_{a}\right\|_{A_{\omega}^{p}} \asymp 1$ for all $a \in \mathbb{D}$. Therefore the assumption yields

$$
1 \asymp\left\|f_{a}\right\|_{A_{\omega}^{p}}^{q} \gtrsim\left\|f_{a}\right\|_{L_{\mu}^{q}}^{q} \gtrsim \frac{\mu(\Delta(a, r))}{(\widehat{\omega}(a)(1-|a|))^{\frac{q}{p}}}, \quad a \in \mathbb{D},
$$

that is, $\mu(\Delta(a, r)) \lesssim(\widehat{\omega}(a)(1-|a|))^{\frac{q}{p}}$ for all $a \in \mathbb{D}$. Since $\omega \in \mathcal{D} \subset \check{\mathcal{D}}$ by the hypothesis, there exists $K=K(\omega)>1$ and $C=C(\omega)>1$ such that $\hat{\omega}(r) \geq C \hat{\omega}\left(1-\frac{1-r}{K}\right)$ for all $0 \leq r<1$ by the definition. Fix now $r=r(K) \in(0,1)$ sufficiently large such that

$$
\Delta(a, r) \supset\left\{t e^{i \theta}:|a| \leq t \leq 1-\frac{1-|a|}{K},|\arg a-\theta| \leq \frac{1-|a|}{2}\left(1-\frac{1}{K}\right)\right\} .
$$

Then, as $\omega \in \mathcal{D} \subset \widehat{\mathcal{D}}$, the right hand inequality in (11) yields

$$
\begin{aligned}
\omega(\Delta(a, r)) & \geq(1-|a|)\left(1-\frac{1}{K}\right)|a| \int_{|a|}^{1-\frac{1-|a|}{K}} \omega(s) d s \\
& \geq(C-1)(1-|a|)\left(1-\frac{1}{K}\right)|a| \widehat{\omega}\left(1-\frac{1-|a|}{K}\right) \\
& \gtrsim \widehat{\omega}(a)(1-|a|)|a|, \quad a \in \mathbb{D},
\end{aligned}
$$

and therefore

$$
\mu(\Delta(a, r)) \lesssim(\widehat{\omega}(a)(1-|a|))^{\frac{q}{p}} \lesssim\left(\frac{\omega(\Delta(a, r))}{|a|}\right)^{\frac{q}{p}}, \quad a \in \mathbb{D} \backslash\{0\} .
$$

The claim (7) now follows from these estimates for all $r=r(\omega) \in(0,1)$ sufficiently large.

To prove (ii), assume first that $I_{d}: A_{\omega}^{p} \rightarrow L_{\mu}^{q}$ is compact. An application of [17, Lemma 2.1] and the right hand inequality in (11) ensure that we may choose $\gamma=\gamma(p, \omega)>0$ sufficiently large such that $\left\|f_{a}\right\|_{A_{\omega}^{p}} \asymp 1$ for all $a \in \mathbb{D}$, and $f_{a} \rightarrow 0$ uniformly on compact subsets of $\mathbb{D}$, as $|a| \rightarrow 1^{-}$. Therefore the closure of the set $\left\{f_{a}: a \in \mathbb{D}\right\}$ is compact in $L_{\mu}^{q}$. Since for each $\varepsilon>0$ the open balls $B\left(f_{a}, \varepsilon\right)=\left\{f \in L_{\mu}^{q}:\left\|f_{a}-f\right\|_{L^{q}}<\varepsilon\right\}$ cover $\overline{\left\{f_{a}: a \in \mathbb{D}\right\}}$, there exists a finite subcover $\left\{B\left(f_{a_{n}}, \varepsilon\right): n=1, \ldots, N=N(\varepsilon)\right\}$. Let now $a \in \mathbb{D}$ be arbitrary, and let $j=j(a) \in\{1, \ldots, N\}$ such that $f_{a} \in B\left(f_{a_{n}}, \varepsilon\right)$. Then, for each $R \in(0,1)$, we have

$$
\begin{aligned}
\int_{\mathbb{D} \backslash D(0, R)}\left|f_{a}(z)\right|^{q} d \mu(z) & \lesssim \int_{\mathbb{D} \backslash D(0, R)}\left|f_{a}(z)-f_{a_{j}}(z)\right|^{q} d \mu(z)+\int_{\mathbb{D} \backslash D(0, R)}\left|f_{a_{j}}(z)\right|^{q} d \mu(z) \\
& \leq\left\|f_{a}-f_{a_{j}}\right\|_{L_{\mu}^{q}}^{q}+\max _{n=1, \ldots, N} \int_{\mathbb{D} \backslash D(0, R)}\left|f_{a_{n}}(z)\right|^{q} d \mu(z) .
\end{aligned}
$$

By fixing $R \in(0,1)$ sufficiently large, and taking into account that $\varepsilon>0$ was arbitrary, we deduce

$$
\lim _{R \rightarrow 1^{-}} \int_{\mathbb{D} \backslash D(0, R)}\left|f_{a}(z)\right|^{q} d \mu(z)=0
$$

uniformly in $a$. This together with the uniform convergence yield 


$$
0=\lim _{|a| \rightarrow 1^{-}}\left\|f_{a}\right\|_{L_{\mu}^{q}}^{q} \geq \lim _{|a| \rightarrow 1^{-}} \int_{\Delta(a, r)}\left|f_{a}(z)\right|^{q} d \mu(z) \gtrsim \lim _{|a| \rightarrow 1^{-}} \frac{\mu(\Delta(a, r))}{(\widehat{\omega}(a)(1-|a|))^{\frac{q}{p}}}
$$

for all $r \in(0,1)$. Now fix $r=r(\omega)$ as in the case (i) to have $\widehat{\omega}(a)(1-|a|) \lesssim \omega(\Delta(a, r))$ for all $a \in \mathbb{D}$. Then we obtain (8).

Conversely, assume (8). Let $\left\{f_{k}\right\}_{k \in \mathbb{N}}$ be a sequence in $A_{\omega}^{p}$ such that $\sup _{k \in \mathbb{N}}\left\|f_{k}\right\|_{A_{\omega}^{p}}=M<\infty$. Then it is easy to see that $\left\{f_{k}\right\}_{k \in \mathbb{N}}$ is uniformly bounded on compact subsets of $\mathbb{D}$ - this follows, for example, from (18) below. Therefore $\left\{f_{k}\right\}_{k \in \mathbb{N}}$ constitutes a normal family by Montel's theorem, and hence we may extract a subsequence $\left\{f_{k_{j}}\right\}_{j \in \mathbb{N}}$ that converges uniformly on compact subsets of $\mathbb{D}$ to a function $f$ which belongs to $\mathcal{H}(\mathbb{D})$ by Weierstrass' theorem. Fatou's lemma now shows that $f \in A_{\omega}^{p}$. For $r \in(0,1)$, fix an $r$-lattice $\left\{a_{n}\right\}_{n \in \mathbb{N}}$. Since $\left|a_{n}\right| \rightarrow 1$, as $n \rightarrow \infty$, we have

$$
\lim _{n \rightarrow \infty} \frac{\mu\left(\Delta\left(a_{n}, r\right)\right)}{\omega\left(\Delta\left(a_{n}, r\right)\right)^{\frac{q}{p}}}=0
$$

by the hypothesis. Therefore, for each $\varepsilon>0$, there exists $N=N(\varepsilon) \in \mathbb{N}$ such that

$$
\frac{\mu\left(\Delta\left(a_{n}, r\right)\right)}{\omega\left(\Delta\left(a_{n}, r\right)\right)^{\frac{q}{p}}}<\varepsilon, \quad n \geq N .
$$

Hence, as in the case (i), Minkowski's inequality in continuous form (Fubini's theorem in the case $q=p$ ), (14), (11) and (10) yield

$$
\begin{aligned}
& \sum_{n=N}^{\infty} \int_{\Delta\left(a_{n}, r\right)}\left|f(z)-f_{k_{j}}(z)\right|^{q} d \mu(z) \\
& \quad \lesssim \sum_{n=N}^{\infty} \int_{\Delta\left(a_{n}, r\right)}\left(\int_{\Delta(z, R)} \frac{\left|f(\zeta)-f_{k_{j}}(\zeta)\right|^{p}}{(1-|\zeta|)^{2}} d A(\zeta)\right)^{\frac{q}{p}} d \mu(z) \\
& \quad \leq \sum_{n=N}^{\infty}\left(\int_{\left\{\zeta: \Delta\left(a_{n}, r\right) \cap \Delta(\zeta, R) \neq \emptyset\right\}} \frac{\left|f(\zeta)-f_{k_{j}}(\zeta)\right|^{p}}{(1-|\zeta|)^{2}} \mu\left(\Delta\left(a_{n}, r\right)\right)^{\frac{p}{q}} d A(\zeta)\right)^{\frac{q}{p}} \\
& \quad \leq \varepsilon \sum_{n=N}^{\infty}\left(\int_{\left\{\zeta: \Delta\left(a_{n}, r\right) \cap \Delta(\zeta, R) \neq \emptyset\right\}} \frac{\left|f(\zeta)-f_{k_{j}}(\zeta)\right|^{p}}{(1-|\zeta|)^{2}} \omega\left(\Delta\left(a_{n}, r\right)\right) d A(\zeta)\right)^{\frac{q}{p}} \\
& \quad \lesssim \varepsilon\left(\sum_{n=N}^{\infty} \int_{\left\{\zeta: \Delta\left(a_{n}, r\right) \cap \Delta(\zeta, R) \neq \emptyset\right\}}\left|f(\zeta)-f_{k_{j}}(\zeta)\right|^{p} \widetilde{\omega}(\zeta) d A(\zeta)\right)^{\frac{q}{p}} \\
& \quad \lesssim \varepsilon\left\|f-f_{k_{j}}\right\|_{A_{\tilde{\omega}}^{q} \asymp \varepsilon\left\|f-f_{k_{j}}\right\|_{A_{\omega}^{p}}^{q} \lesssim M^{q} \varepsilon .}
\end{aligned}
$$

Since

$$
\lim _{j \rightarrow \infty} \sum_{n=1}^{N-1} \int_{\Delta\left(a_{n}, r\right)}\left|f(z)-f_{k_{j}}(z)\right|^{q} d \mu(z)=0
$$

by the uniform convergence in compact subsets, we deduce 


$$
\begin{aligned}
\limsup _{j \rightarrow \infty} \int_{\mathbb{D}}\left|f(z)-f_{k_{j}}(z)\right|^{q} d \mu(z) \leq & \limsup _{j \rightarrow \infty}\left(\sum_{n=1}^{N-1} \int_{\Delta\left(a_{n}, r\right)}\left|f(z)-f_{k_{j}}(z)\right|^{q} d \mu(z)\right. \\
& \left.+\sum_{n=N}^{\infty} \int_{\Delta\left(a_{n}, r\right)}\left|f(z)-f_{k_{j}}(z)\right|^{q} d \mu(z)\right) \lesssim \varepsilon .
\end{aligned}
$$

Since $\varepsilon>0$ was arbitrary, we have

$$
\limsup _{j \rightarrow \infty} \int_{\mathbb{D}}\left|f(z)-f_{k_{j}}(z)\right|^{q} d \mu(z)=0,
$$

and hence $I_{d}: A_{\omega}^{p} \rightarrow L_{\mu}^{q}$ is compact. This completes the proof of the theorem.

\section{Proof of Theorem 1}

With the auxiliary results proved in the previous sections we are ready for the proof of the main result. We will follow the arguments used in [1] with appropriate modifications. The following two propositions will prove Theorem 1 . The first one gives necessary conditions for $u C_{\varphi}-v C_{\psi}: A_{\omega}^{p} \rightarrow A_{\nu}^{q}$ to be compact.

Proposition 1 Let $\omega, v \in \mathcal{D}, 0<p, q<\infty, u, v \in \mathcal{H}(\mathbb{D})$ and $\varphi$ and $\psi$ be analytic self-maps of $\mathbb{D}$. If $u C_{\varphi}-v C_{\psi}: A_{\omega}^{p} \rightarrow A_{v}^{q}$ is compact, then

$$
\lim _{|z| \rightarrow 1^{-}}\left|\delta_{1}(z)\right|\left(|u(z)| \frac{(\widehat{v}(z)(1-|z|))^{\frac{1}{q}}}{(\widehat{\omega}(\varphi(z))(1-|\varphi(z)|))^{\frac{1}{p}}}+|v(z)| \frac{(\widehat{v}(z)(1-|z|))^{\frac{1}{q}}}{(\widehat{\omega}(\psi(z))(1-|\psi(z)|))^{\frac{1}{p}}}\right)=0
$$

and there exits $\gamma=\gamma(\omega, p)>0$ such that

$$
\lim _{|z| \rightarrow 1^{-}}\left(\frac{\left|1-\bar{\varphi}(z) \delta_{1}(z)\right|^{\gamma}}{(\widehat{\omega}(\varphi(z))(1-|\varphi(z)|))^{\frac{1}{p}}}+\frac{\left|1-\bar{\psi}(z) \delta_{2}(z)\right|^{\gamma}}{(\widehat{\omega}(\psi(z))(1-|\psi(z)|))^{\frac{1}{p}}}\right)|u(z)-v(z)|(\widehat{v}(z)(1-|z|))^{\frac{1}{q}}=0 .
$$

Proof Consider the test functions $f_{a}$ defined in (15), and set $F_{a}(z)=\varphi_{a}(z) f_{a}(z)$ for all $a, z \in \mathbb{D}$. Obviously, $\left\|F_{a}\right\|_{A_{\omega}^{p}} \leq\left\|f_{a}\right\|_{A_{\omega}^{p}}$ for all $a \in \mathbb{D}$. Further, by the Proof of Theorem 2, both $f_{a}$ and $F_{a}$ tend to zero uniformly on compact subsets of $\mathbb{D}$ as $|a| \rightarrow 1^{-}$, and $\left\|f_{a}\right\|_{A_{\omega}^{p}} \asymp 1$ for all $a \in \mathbb{D}$ if $\gamma=\gamma(\omega, p)>0$ is sufficiently large. Since $u C_{\varphi}-v C_{\psi}: A_{\omega}^{p} \rightarrow A_{\nu}^{q}$ is compact by the hypothesis, we therefore have

$$
\lim _{|a| \rightarrow 1^{-}}\left\|u C_{\varphi}\left(f_{a}\right)-v C_{\psi}\left(f_{a}\right)\right\|_{A_{v}^{q}}=0
$$

and

$$
\lim _{|a| \rightarrow 1^{-}}\left\|u C_{\varphi}\left(F_{a}\right)-v C_{\psi}\left(F_{a}\right)\right\|_{A_{\nu}^{q}}=0 .
$$

Also, if $\lim _{|a| \rightarrow 1^{-}}$is replaced by $\sup _{a \in \mathbb{D}}$ in the above formulas, then the corresponding quantities are bounded.

We next observe that for each $\omega \in \widehat{\mathcal{D}}$ and $0<q<\infty$, there exists a positive bounded function $C_{\omega}$ on $[0,1)$ such that 


$$
|f(z)| \leq \frac{C_{\omega}(|z|)}{(\widehat{\omega}(z)(1-|z|))^{\frac{1}{q}}}\|f\|_{A_{\omega}^{q}}, \quad f \in A_{\omega}^{q}, \quad z \in \mathbb{D},
$$

and $C_{\omega}(z) \rightarrow 0$ as $|z| \rightarrow 1^{-}$. Namely, for each $z \in \mathbb{D}$ we have

$$
\begin{aligned}
\|f\|_{A_{\omega}^{q}}^{q} & \geq \int_{\mathbb{D} \backslash D\left(0, \frac{1+|z|}{2}\right)}|f(\zeta)|^{q} \omega(\zeta) d A(\zeta) \geq M_{q}^{q}\left(\frac{1+|z|}{2}, f\right) \int_{\frac{1+|z|}{2}}^{1} r \omega(r) d r \\
& \geq \frac{1}{2} M_{q}^{q}\left(\frac{1+|z|}{2}, f\right) \widehat{\omega}\left(\frac{1+|z|}{2}\right) \gtrsim M_{q}^{q}\left(\frac{1+|z|}{2}, f\right) \widehat{\omega}(z),
\end{aligned}
$$

which combined with the well-known inequality $M_{\infty}(|z|, f) \lesssim M_{q}\left(\frac{1+|z|}{2}, f\right)(1-|z|)^{-\frac{1}{q}}$, valid for all $f \in \mathcal{H}(\mathbb{D})$, yields (18) because

$$
\int_{\mathbb{D} \backslash D\left(0, \frac{1+|z|}{2}\right)}|f(\zeta)|^{q} \omega(\zeta) d A(\zeta) \rightarrow 0, \quad|z| \rightarrow 1^{-},
$$

for each $f \in A_{\omega}^{q}$.

By combining (16) and (17) with (18), we deduce

$$
\lim _{\max (|a|,|z|) \rightarrow 1^{-}}\left|u C_{\varphi}\left(f_{a}\right)(z)-v C_{\psi}\left(f_{a}\right)(z)\right|(\widehat{v}(z)(1-|z|))^{\frac{1}{q}}=0
$$

and

$$
\lim _{\max (|a|,|z|) \rightarrow 1^{-}}\left|u C_{\varphi}\left(F_{a}\right)(z)-v C_{\psi}\left(F_{a}\right)(z)\right|(\widehat{v}(z)(1-|z|))^{\frac{1}{q}}=0 .
$$

By choosing $a=\varphi(z)$, we obtain

$$
\lim _{|z| \rightarrow 1^{-}}\left|u(z) f_{\varphi(z)}(\varphi(z))-v(z) f_{\varphi(z)}(\psi(z))\right|(\widehat{v}(z)(1-|z|))^{\frac{1}{q}}=0
$$

and

$$
\lim _{|z| \rightarrow 1^{-}}\left|\delta_{1}(z)\right||v(z)|\left|f_{\varphi(z)}(\psi(z))\right|(\widehat{v}(z)(1-|z|))^{\frac{1}{q}}=0 .
$$

Since $\left|\delta_{1}(z)\right|<1$ for all $z \in \mathbb{D}$, and

$$
|u(z)|\left|f_{\varphi(z)}(\varphi(z))\right| \leq\left|u(z) f_{\varphi(z)}(\varphi(z))-v(z) f_{\varphi(z)}(\psi(z))\right|+|v(z)|\left|f_{\varphi(z)}(\psi(z))\right|,
$$

by combining (19) and (20) we deduce

$$
\begin{aligned}
& \lim _{|z| \rightarrow 1^{-}} \frac{\left|\delta_{1}(z)\right||u(z)|(\widehat{v}(z)(1-|z|))^{\frac{1}{q}}}{(\widehat{\omega}(\varphi(z))(1-|\varphi(z)|))^{\frac{1}{p}}} \\
& \quad=\lim _{|z| \rightarrow 1^{-}}\left|\delta_{1}(z)\right||u(z)|\left|f_{\varphi(z)}(\varphi(z))\right|(\widehat{v}(z)(1-|z|))^{\frac{1}{q}}=0 .
\end{aligned}
$$

Further, we claim that for each $0<\gamma<\infty$ and each bounded set $\Omega \subset \mathbb{C}$, there exists a constant $C=C(\gamma, \Omega)>0$ such that 


$$
\left|1-z^{\gamma}\right| \leq C|1-z|, \quad z \in \Omega,
$$

the proof of which is postponed for a moment. By using this and the fact that $1-\frac{1-|a|^{2}}{1-\bar{a} b}=\bar{a} \varphi_{a}(b)$ for all $a, b \in \mathbb{D}$, we deduce

$$
\begin{aligned}
\left|f_{\varphi(z)}(\varphi(z))-f_{\varphi(z)}(\psi(z))\right| & =\left|f_{\varphi(z)}(\varphi(z))\right|\left|1-\left(\frac{1-|\varphi(z)|^{2}}{1-\overline{\varphi(z)} \psi(z)}\right)^{\gamma}\right| \\
& \lesssim\left|f_{\varphi(z)}(\varphi(z))\right|\left|1-\frac{1-|\varphi(z)|^{2}}{1-\overline{\varphi(z)} \psi(z)}\right| \leq\left|f_{\varphi(z)}(\varphi(z))\right|\left|\delta_{1}(z)\right|,
\end{aligned}
$$

and hence

$$
\begin{aligned}
|u(z)-v(z)|\left|f_{\varphi(z)}(\psi(z))\right| \leq & \left|u(z) f_{\varphi(z)}(\varphi(z))-v(z) f_{\varphi(z)}(\psi(z))\right| \\
& +|u(z)|\left|f_{\varphi(z)}(\varphi(z))-f_{\varphi(z)}(\psi(z))\right| \\
\lesssim & \left|u(z) f_{\varphi(z)}(\varphi(z))-v(z) f_{\varphi(z)}(\psi(z))\right| \\
& +\left|\delta_{1}(z)\right||u(z)|\left|f_{\varphi(z)}(\varphi(z))\right|, \quad z \in \mathbb{D} .
\end{aligned}
$$

Therefore, by (19) and (21), we finally obtain

$$
\begin{aligned}
& \lim _{|z| \rightarrow 1^{-}}\left(\frac{\left|1-\bar{\varphi}(z) \delta_{1}(z)\right|^{\gamma}}{(\widehat{\omega}(\varphi(z))(1-|\varphi(z)|))^{\frac{1}{p}}}\right)|u(z)-v(z)|(\widehat{v}(z)(1-|z|))^{\frac{1}{q}} \\
& =\lim _{|z| \rightarrow 1^{-}}|u(z)-v(z)|\left|f_{\varphi(z)}(\psi(z))\right|(\widehat{v}(z)(1-|z|))^{\frac{1}{q}}=0 .
\end{aligned}
$$

By following the reasoning above, but with the choice $a=\psi(z)$, we obtain

$$
\lim _{|z| \rightarrow 1^{-}} \frac{\left|\delta_{1}(z)\right||v(z)|(\widehat{v}(z)(1-|z|))^{\frac{1}{q}}}{(\widehat{\omega}(\psi(z))(1-|\psi(z)|))^{\frac{1}{p}}}=0
$$

as an analogue of (21), and then eventually

$$
\lim _{|z| \rightarrow 1^{-}}\left(\frac{\left|1-\bar{\psi}(z) \delta_{2}(z)\right|^{\gamma}}{(\widehat{\omega}(\psi(z))(1-|\psi(z)|))^{\frac{1}{p}}}\right)|u(z)-v(z)|(\widehat{v}(z)(1-|z|))^{\frac{1}{q}}=0 .
$$

Therefore to finish the proof of the proposition, it remains to establish (22). If $z \in \Omega \backslash\{z:|1-z|<1 / 2\}$, then

$$
\left|1-z^{\gamma}\right| \leq 1+\sup _{z \in \Omega}|z|^{\gamma} \leq 2\left(1+\sup _{z \in \Omega}|z|^{\gamma}\right)|1-z|,
$$

while if $z \in \Omega \cap\{z:|1-z|<1 / 2\}$, we have

$$
\left|1-z^{\gamma}\right|=\left|\int_{z}^{1} \gamma \zeta^{\gamma-1} d \zeta\right| \leq \gamma \int_{z}^{1}|\zeta|^{\gamma-1}|d \zeta| \leq \frac{\gamma \max \left\{1,3^{\gamma-1}\right\}}{2^{\gamma-1}}|1-z| .
$$

This proves (22), and completes the proof of the proposition. 
Sufficient conditions for the compactness of $u C_{\varphi}-v C_{\psi}: A_{\omega}^{p} \rightarrow A_{v}^{q}$ are given in the next result.

Proposition 2 Let $\omega, v \in \mathcal{D}$ and $0<p \leq q<\infty$ such that $\widehat{v}(z)(1-|z|) \lesssim(\widehat{\omega}(z)(1-|z|))^{\frac{q}{p}}$ for all $z \in \mathbb{D}$. Further, let $u, v \in \mathcal{H}(\mathbb{D})$ and $\varphi$ and $\psi$ analytic self-maps of $\mathbb{D}$ such that $(4)$ is satisfied. If there exists $\gamma>0$ such that (5) and (6) are satisfied, then $u C_{\varphi}-v C_{\psi}: A_{\omega}^{p} \rightarrow A_{v}^{q}$ is compact.

Proof It suffices to show that for any norm bounded sequence $\left\{f_{n}\right\}$ in $A_{\omega}^{p}$ which tends to zero uniformly on compact subsets of $\mathbb{D}$ as $n \rightarrow \infty$, we have $\left\|\left(u C_{\varphi}-v C_{\psi}\right)\left(f_{n}\right)\right\|_{A_{\nu}^{q} \rightarrow 0}$ as $n \rightarrow \infty$. For simplicity, assume $\left\|f_{n}\right\|_{A_{\omega}^{p}} \leq 1$ for all $n$. Fix $0<r<R<1$, and denote $E=\left\{z \in \mathbb{D}:\left|\delta_{1}(z)\right|<r\right\}$ and $E^{\prime}=\mathbb{D} \backslash E$. Write

$$
\left(u C_{\varphi}-v C_{\psi}\right)\left(f_{n}\right)=\left(u C_{\varphi}-v C_{\psi}\right)\left(f_{n}\right) \chi_{E^{\prime}}+(u-v) C_{\psi}\left(f_{n}\right) \chi_{E}+u\left(C_{\varphi}-C_{\psi}\right)\left(f_{n}\right) \chi_{E},
$$

and observe that it is enough to prove that each of the three quantities

$$
\left\|\left(u C_{\varphi}-v C_{\psi}\right)\left(f_{n}\right) \chi_{E^{\prime}}\right\|_{A_{v}^{q}}, \quad\left\|(u-v) C_{\psi}\left(f_{n}\right) \chi_{E}\right\|_{A_{v}^{q}} \quad \text { and } \quad\left\|u\left(C_{\varphi}-C_{\psi}\right)\left(f_{n}\right) \chi_{E}\right\|_{A_{v}^{q}}
$$

tends to zero as $n \rightarrow \infty$.

We begin with considering the first two quantities in (23). By the definition of the set $E$ we have the estimates

$$
\left|\left(u C_{\varphi}-v C_{\psi}\right)\left(f_{n}\right) \chi_{E^{\prime}}\right| \leq \frac{1}{r}\left(\left|\delta_{1} u C_{\varphi}\left(f_{n}\right)\right|+\left|\delta_{1} v C_{\psi}\left(f_{n}\right)\right|\right)
$$

and

$$
\left|(u-v) C_{\psi}\left(f_{n}\right) \chi_{E}\right| \leq\left(\frac{1}{1-r}\right)^{\gamma}\left|1-\bar{\psi} \delta_{2}\right|^{\gamma}|u-v|\left|C_{\psi}\left(f_{n}\right)\right|
$$

on $\mathbb{D}$. Therefore it suffices to prove that $\delta_{1} u C_{\varphi}, \delta_{1} v C_{\psi}$ and $\left(1-\bar{\psi} \delta_{2}\right)^{\gamma}(u-v) C_{\psi}$ are compact operators from $A_{\omega}^{p}$ to $L_{\nu}^{q}$. We show in detail that $\delta_{1} u C_{\varphi}$ is compact - the same argument shows the compactness of the other two operators.

Let $\mu$ be a finite nonnegative Borel measure on $\mathbb{D}$ and $h$ a measureable function on $\mathbb{D}$. For an analytic self-map $\varphi$ of $\mathbb{D}$, the weighted pushforward measure is defined by

$$
\varphi_{*}(h, \mu)(M)=\int_{\varphi^{-1}(M)} h d \mu
$$

for each measurable set $M \subset \mathbb{D}$. If $\mu$ is the Lebesgue measure, we omit the measure in the notation and write $\varphi_{*}(h)(M)$ for the left hand side of (24). By the measure theoretic change of variable [9, Section 39], we have $\left\|\delta_{1} u C_{\varphi}(f)\right\|_{L_{v}^{q}}=\|f\|_{L_{\varphi_{*}\left(\left|\delta_{1} u\right| q_{v}\right)}^{q}}$ for each $f \in A_{\omega}^{p}$. Therefore Theorem 2 shows that $\delta_{1} u C_{\varphi}: A_{\omega}^{p} \rightarrow L_{\nu}^{q}$ is compact if and only if

$$
\frac{\varphi_{*}\left(\left|\delta_{1} u\right|^{q} \nu\right)(\Delta(a, r))}{\omega(\Delta(a, r))^{\frac{q}{p}}}=\frac{\int_{\varphi^{-1}(\Delta(a, r))}\left|\delta_{1}(z) u(z)\right|^{q} \nu(z) d A(z)}{\omega(\Delta(a, r))^{\frac{q}{p}}} \rightarrow 0, \quad|a| \rightarrow 1^{-} .
$$

This is what we prove next. Define 


$$
W_{a, r}=\sup _{z \in \varphi^{-1}(\Delta(a, r))}\left|\left(\delta_{1}(z) u(z)\right)^{q} \frac{\widehat{v}(z)(1-|z|)}{(\widehat{\omega}(\varphi(z))(1-|\varphi(z)|))^{\frac{q}{p}}}\right| .
$$

Then $W_{a, r} \rightarrow 0$, as $|a| \rightarrow 1^{-}$, by the hypothesis (5). Moreover, for $a \in \mathbb{D}$ and $z \in \varphi^{-1}(\Delta(a, r)),(11)$ yields

$$
\left|\delta_{1}(z) u(z)\right|^{q} \lesssim W_{a, r} \frac{(\widehat{\omega}(a)(1-|a|))^{\frac{q}{p}}}{\widehat{v}(z)(1-|z|)}
$$

and therefore, for each $\varepsilon \in(0,1)$ we have

$$
\begin{aligned}
& \varphi_{*}\left(\left|\delta_{1} u\right|^{q} \nu\right)(\Delta(a, r))= \int_{\varphi^{-1}(\Delta(a, r))}\left|\delta_{1}(z) u(z)\right|^{q} \nu(z) d A(z) \\
& \lesssim \sup _{z \in \mathbb{D}}\left|\left(\delta_{1}(z) u(z)\right)^{q} \frac{\widehat{v}(z)(1-|z|)}{(\widehat{\omega}(z)(1-|z|))^{\frac{q}{p}}}\right|^{1-\varepsilon} W_{a, r}^{\varepsilon}(\widehat{\omega}(a)(1-|a|))^{\varepsilon \frac{q}{p}} \\
& \cdot \int_{\varphi^{-1}(\Delta(a, r))} \frac{(\widehat{\omega}(z)(1-|z|))^{(1-\varepsilon)^{\frac{q}{p}}} \nu(z)}{\widehat{v}(z)(1-|z|)} d A(z), \quad a \in \mathbb{D} .
\end{aligned}
$$

Before proceeding further, we indicate how to get to this point with the operator $\left(1-\bar{\psi} \delta_{2}\right)^{\gamma}(u-v) C_{\psi}$. After the measure theoretic change of variable and an application of Theorem 2, consider

$$
V_{a, r}=\sup _{z \in \psi^{-1}(\Delta(a, r))}\left|\left(1-\bar{\psi}(z) \delta_{2}(z)\right)^{\gamma q}(u(z)-v(z))^{q} \frac{\widehat{v}(z)(1-|z|)}{(\widehat{\omega}(\psi(z))(1-|\psi(z)|))^{\frac{q}{p}}}\right|
$$

instead of $W_{a, r}$. Then $V_{a, r} \rightarrow 0$, as $|a| \rightarrow 1^{-}$, by the hypothesis (6), and moreover, for $a \in \mathbb{D}$ and $z \in \psi^{-1}(\Delta(a, r)),(11)$ yields

$$
\left|1-\bar{\psi}(z) \delta_{2}(z)\right|^{\gamma q}|u(z)-v(z)|^{q} \lesssim V_{a, r} \frac{(\widehat{\omega}(a)(1-|a|))^{\frac{q}{p}}}{\widehat{v}(z)(1-|z|)} .
$$

Therefore, for each $\varepsilon \in(0,1)$, we have

$$
\begin{aligned}
& \psi_{*}\left(\left|1-\bar{\psi} \delta_{2}\right|^{\gamma q}|u-v|^{q} v\right)(\Delta(a, r)) \\
& \lesssim \sup _{z \in \mathbb{D}}\left|\left(1-\bar{\psi}(z) \delta_{2}(z)\right)^{\gamma q}(u(z)-v(z))^{q} \frac{\widehat{v}(z)(1-|z|)}{(\widehat{\omega}(z)(1-|z|))^{\frac{q}{p}}}\right|^{1-\varepsilon} V_{a, r}^{\varepsilon}(\widehat{\omega}(a)(1-|a|))^{\varepsilon^{\frac{q}{p}}} \\
& \quad \cdot \int_{\psi^{-1}(\Delta(a, r))} \frac{(\widehat{\omega}(z)(1-|z|))^{(1-\varepsilon) \frac{q}{p}} \nu(z)}{\widehat{v}(z)(1-|z|)} d A(z), \quad a \in \mathbb{D} .
\end{aligned}
$$

To estimate this last integral, which is the same as the one appearing in (25), we first show that there exists $\varepsilon=\varepsilon(\omega, v, q, p) \in(0,1)$ sufficiently small such that the function 


$$
\mu(z)=\mu_{\omega, v, \varepsilon, q, p}(z)=\frac{(\widehat{\omega}(z)(1-|z|))^{(1-\varepsilon)^{\frac{q}{p}}} \nu(z)}{\widehat{v}(z)(1-|z|)}, \quad z \in \mathbb{D},
$$

is a weight and belongs to $\mathcal{D}$. To see that $\mu \in \widehat{\mathcal{D}}$, for $n \in \mathbb{N} \cup\{0\}$, define $\rho_{n}$ by $\widehat{v}\left(\rho_{n}\right)=\frac{\widehat{v}(0)}{K^{n}}$, where $K>1$. Let $0 \leq r<1$ and fix $M \in \mathbb{N} \cup\{0\}$ such that $\rho_{M} \leq r<\rho_{M+1}$. Set $\alpha=(1-\varepsilon) \frac{q}{p}$ for short. Since $\omega, v \in \mathcal{D}$ by the hypothesis, (11) yields

$$
\begin{aligned}
\widehat{\omega}\left(\rho_{j+N+1}\right) & \leq \widehat{\omega}\left(\rho_{j}\right) \lesssim \widehat{\omega}\left(\rho_{j+N+1}\right)\left(\frac{1-\rho_{j}}{1-\rho_{j+N+1}}\right)^{\beta(\omega)} \lesssim \widehat{\omega}\left(\rho_{j+N+1}\right)\left(\frac{\widehat{v}\left(\rho_{j}\right)}{\widehat{v}\left(\rho_{j+N+1}\right)}\right)^{\frac{\beta(\omega)}{\alpha(v)}} \\
& =\widehat{\omega}\left(\rho_{j+N+1}\right) K^{(N+1) \frac{\beta(\omega)}{\alpha(v)}} \asymp \widehat{\omega}\left(\rho_{j+N+1}\right)
\end{aligned}
$$

and $1-\rho_{j} \asymp 1-\rho_{j+1}$ for all $j$ and for each fixed $N \in \mathbb{N}$. Therefore

$$
\begin{aligned}
\int_{r}^{1} \frac{(\widehat{\omega}(t))^{\alpha}}{(1-t)^{1-\alpha}} \frac{v(t)}{\widehat{v}(t)} d t & \leq \sum_{j=M}^{\infty} \int_{\rho_{j}}^{\rho_{j+1}} \frac{(\widehat{\omega}(t))^{\alpha}}{(1-t)^{1-\alpha}} \frac{v(t)}{\widehat{v}(t)} d t \leq \sum_{j=M}^{\infty} \frac{\left(\widehat{\omega}\left(\rho_{j}\right)\right)^{\alpha}}{\left(1-\rho_{j+1}\right)^{1-\alpha}} \int_{\rho_{j}}^{\rho_{j+1}} \frac{v(t)}{\widehat{v}(t)} d t \\
& =\sum_{j=M}^{\infty} \frac{\left(\widehat{\omega}\left(\rho_{j}\right)\right)^{\alpha}}{\left(1-\rho_{j+1}\right)^{1-\alpha}} \int_{\rho_{j+N}}^{\rho_{j+N+1}} \frac{v(t)}{\widehat{v}(t)} d t \asymp \int_{\rho_{M+N}}^{1} \frac{(\widehat{\omega}(t))^{\alpha}}{(1-t)^{1-\alpha}} \frac{v(t)}{\widehat{v}(t)} d t .
\end{aligned}
$$

Another application of (11) shows that there exists $N=N(v) \in \mathbb{N}$ such that $\rho_{M+N} \geq \frac{1+\rho_{M+1}}{2}$. Namely, the right hand inequality implies

$$
\frac{1-\rho_{M+1}}{1-\rho_{M+N}} \geq\left(\frac{\widehat{v}\left(\rho_{M+1}\right)}{C \widehat{v}\left(\rho_{M+N}\right)}\right)^{\frac{1}{\beta}}=\left(\frac{K^{N-1}}{C}\right)^{\frac{1}{\beta}} \geq 2
$$

for sufficiently large $N$ giving what we want. Therefore

$$
\int_{r}^{1} \frac{(\widehat{\omega}(t))^{\alpha}}{(1-t)^{1-\alpha}} \frac{v(t)}{\widehat{v}(t)} d t \lesssim \int_{\frac{1+\rho_{M+1}}{2}}^{1} \frac{(\widehat{\omega}(t))^{\alpha}}{(1-t)^{1-\alpha}} \frac{v(t)}{\widehat{v}(t)} d t \leq \int_{\frac{1+r}{2}}^{1} \frac{(\widehat{\omega}(t))^{\alpha}}{(1-t)^{1-\alpha}} \frac{v(t)}{\widehat{v}(t)} d t,
$$

which shows that $\mu \in \widehat{\mathcal{D}}$, provided $\mu$ is a weight.

We next show that $\mu$ is a weight in $\check{\mathcal{D}}$. By using (11), with $\beta$ in place of $\alpha$, we obtain

$$
\begin{aligned}
\int_{r}^{1} \frac{(\widehat{\omega}(t))^{\alpha}}{(1-t)^{1-\alpha}} \frac{v(t)}{\widehat{v}(t)} d t & \lesssim \int_{r}^{1} \frac{\left(\widehat{\omega}(r)\left(\frac{1-t}{1-r}\right)^{\beta}(1-t)\right)^{\alpha}}{\widehat{v}(t)(1-t)} v(t) d t \\
& \lesssim \frac{\widehat{\omega}(r)^{\alpha}}{(1-r)^{\beta \alpha}} \int_{r}^{1} \frac{(1-t)^{(1+\beta) \alpha}}{\widehat{v}(t)(1-t)} v(t) d t
\end{aligned}
$$

Now fix $\varepsilon \in(0,1)$ sufficient small such that $\sigma=(1+\beta) \alpha=(1+\beta)(1-\varepsilon) \frac{q}{p}>1$. By $[19$, Lemma 3], the last expression above is dominated by a constant times

$$
\frac{\widehat{\omega}(r)^{\alpha}}{(1-r)^{\beta \alpha}}(1-r)^{\sigma-1}=\frac{(\widehat{\omega}(r)(1-r))^{\alpha}}{1-r} .
$$

Since $v \in \check{\mathcal{D}}$ by the hypothesis, there exists $K=K(v)>1$ such that 


$$
\begin{aligned}
\frac{(\widehat{\omega}(r)(1-r))^{\alpha}}{1-r} & \asymp \frac{(\widehat{\omega}(r)(1-r))^{\alpha}}{\widehat{v}(r)(1-r)} \int_{r}^{1-\frac{1-r}{K}} v(t) d t \\
& \asymp \int_{r}^{1-\frac{1-r}{K}} \frac{(\widehat{\omega}(t)(1-t))^{\alpha}}{\widehat{v}(t)(1-t)} v(t) d t,
\end{aligned}
$$

where the last step is a consequence of (11), applied to both weights $\omega, \nu \in \mathcal{D}$. This reasoning shows that $\mu$ is a weight in $\check{\mathcal{D}}$, and thus $\mu \in \mathcal{D}$.

We return to estimate the last integral in (25). By [16, Proposition 18], the operator $C_{\varphi}: A_{\mu}^{p} \rightarrow A_{\mu}^{p}$ is bounded for each $0<p<\infty$. By the measure theoretic change of variable, this is equivalent to saying that $I_{d}: A_{\mu}^{p} \rightarrow L_{\varphi_{*}(\mu)}^{p}$ is bounded. Since we just proved that $\mu \in \mathcal{D}$, this is in turn equivalent to $\varphi_{*}(\mu)(\Delta(a, r)) \lesssim \mu(\Delta(a, r))$ by Theorem 2 . By the definition of these two measures and the poof of the fact $\mu \in \check{\mathcal{D}}$ above, we have

$$
\int_{\varphi^{-1}(\Delta(a, r))} \mu(z) d A(z) \lesssim \int_{\Delta(a, r)} \mu(z) d A(z) \lesssim(\widehat{\omega}(a)(1-|a|))^{(1-\varepsilon) \frac{q}{p}}, \quad a \in \mathbb{D} .
$$

This combined with (25) gives

$$
\varphi_{*}\left(\left|\delta_{1} u\right|^{q} \nu\right)(\Delta(a, r)) \lesssim \sup _{z \in \mathbb{D}}\left|\left(\delta_{1}(z) u(z)\right)^{q} \frac{\widehat{v}(z)(1-|z|)}{(\widehat{\omega}(z)(1-|z|))^{\frac{q}{p}}}\right|^{1-\varepsilon} W_{a, r}^{\varepsilon}(\widehat{\omega}(a)(1-|a|))^{\frac{q}{p}} .
$$

Since the supremum above is bounded by the hypothesis (4), and $\widehat{\omega}(a)(1-|a|) \lesssim \omega(\Delta(a, r))$ for $r=r(\omega) \in(0,1)$ sufficiently large by the Proof of Theorem 2, we deduce via Theorem 2 that $\delta_{1} u C_{\varphi}: A_{\omega}^{p} \rightarrow L_{v}^{q}$ is compact. As mentioned already, $\delta_{1} v C_{\psi}$ and $\left(1-\bar{\psi} \delta_{2}\right)^{\gamma}(u-v) C_{\psi}$ can be treated in the same way.

It remains to deal with the third term in (23). By Lemma 1, Fubini's theorem and (11), we have

$$
\begin{aligned}
\left\|u\left(C_{\varphi}-C_{\psi}\right)\left(f_{n}\right) \chi_{E}\right\|_{A_{v}^{q}}^{q}= & \int_{E}|u(z)|^{q}\left|f_{n}(\varphi(z))-f_{n}(\psi(z))\right|^{q} \nu(z) d A(z) \\
& \lesssim \int_{E} \frac{\left|u(z) \delta_{1}(z)\right|^{q}}{(\widehat{\omega}(\varphi(z))(1-|\varphi(z)|))^{\frac{q}{p}}} \int_{(\varphi(z), R)}\left|f_{n}(\zeta)\right|^{p} \widetilde{\omega}(\zeta) d A(\zeta) \nu(z) d A(z) \\
\leq & \int_{\mathbb{D}}\left|f_{n}(\zeta)\right|^{p} \widetilde{\omega}(\zeta) \\
& \left.\cdot \int_{\varphi^{-1}(\Delta(\zeta, R)) \cap E} \frac{\left|u(z) \delta_{1}(z)\right|^{q}}{(\widehat{\omega}(\varphi(z))(1-|\varphi(z)|))^{\frac{q}{p}}} v(z) d A(z)\right) d A(\zeta) \\
& =\int_{\mathbb{D}}\left|f_{n}(\zeta)\right|^{p} \widetilde{\omega}(\zeta)\left(\int_{\varphi^{-1}(\Delta(\zeta, R))} \frac{\left|u(z) \delta_{1}(z)\right|^{q}}{(\widehat{\omega}(\zeta)(1-|\zeta|))^{\frac{q}{p}}} v(z) d A(z)\right) d A(\zeta) \\
= & \int_{\mathbb{D}}\left|f_{n}(\zeta)\right|^{p} \frac{\varphi_{*}\left(\left|\delta_{1} u\right|^{q} \nu\right)(\Delta(\zeta, R))}{(\widehat{\omega}(\zeta)(1-|\zeta|))^{\frac{q}{p}}} \widetilde{\omega}(\zeta) d A(\zeta) .
\end{aligned}
$$

Since the identity operator from $A_{\omega}^{p}$ to $L_{\varphi_{*}\left(\left|\delta_{1} u\right|^{q} \nu\right)}^{q}$ is compact, it is also bounded. This and Theorem 2 yield 


$$
\begin{aligned}
\left\|u\left(C_{\varphi}-C_{\psi}\right)\left(f_{n}\right) \chi_{E}\right\|_{A_{v}^{q}}^{q} \lesssim & \sup _{\zeta \in D(0, r)} \frac{\varphi_{*}\left(\left|\delta_{1} u\right|^{q} \nu\right)(\Delta(\zeta, R))}{(\widehat{\omega}(\zeta)(1-|\zeta|))^{\frac{q}{p}}} \int_{D(0, r)}\left|f_{n}(\zeta)\right|^{p} \widetilde{\omega}(\zeta) d A(\zeta) \\
& +\sup _{\zeta \in \mathbb{D} \backslash D(0, r)} \frac{\varphi_{*}\left(\left|\delta_{1} u\right|^{q} \nu\right)(\Delta(\zeta, R))}{(\widehat{\omega}(\zeta)(1-|\zeta|))^{\frac{q}{p}}} \int_{\mathbb{D} \backslash D(0, r)}\left|f_{n}(\zeta)\right|^{p} \widetilde{\omega}(\zeta) d A(\zeta) \\
& \lesssim \sup _{\zeta \in D(0, r)}\left|f_{n}(\zeta)\right|^{p}+\sup _{\zeta \in \mathbb{D} \backslash D(0, r)} \frac{\varphi_{*}\left(\left|\delta_{1} u\right|^{q} \nu\right)(\Delta(\zeta, R))}{(\widehat{\omega}(\zeta)(1-|\zeta|))^{\frac{q}{p}}}, \quad 0<r<1 .
\end{aligned}
$$

By choosing $0<r<1$ sufficiently large, the last term can be made smaller than a pregiven $\varepsilon>0$. For such fixed $r$, the first term tends to zero as $n \rightarrow \infty$ by the uniform convergence. Therefore

$$
\left\|u\left(C_{\varphi}-C_{\psi}\right)\left(f_{n}\right) \chi_{E}\right\|_{A_{v}^{q}}^{q} \rightarrow 0, \quad n \rightarrow \infty
$$

and hence also the last term in (23) tends to zero. This finishes the proof of the proposition.

Funding Open access funding provided by University of Eastern Finland (UEF) including Kuopio University Hospital.

Open Access This article is licensed under a Creative Commons Attribution 4.0 International License, which permits use, sharing, adaptation, distribution and reproduction in any medium or format, as long as you give appropriate credit to the original author(s) and the source, provide a link to the Creative Commons licence, and indicate if changes were made. The images or other third party material in this article are included in the article's Creative Commons licence, unless indicated otherwise in a credit line to the material. If material is not included in the article's Creative Commons licence and your intended use is not permitted by statutory regulation or exceeds the permitted use, you will need to obtain permission directly from the copyright holder. To view a copy of this licence, visit http://creativecommons.org/licenses/by/4.0/.

\section{References}

1. Acharyya, S., Wu, Z.: Compact and Hilbert-Schmidt differences of weighted composition operators. Int. Equ. Operator Theory 88, 465-482 (2017)

2. Bonet, J., Lindström, M., Wolf, E.: Differences of composition operators between weighted Banach spaces of holomorphic functions. J. Aust. Math. Soc. 84, 9-20 (2008)

3. Choe, B.R., Choi, K., Koo, H., Yang, J.: Difference of weighted composition operators. J. Funct. Anal. 278, 108401 (2020). 38 pp

4. Contreras, M.D., Hernández-Díaz, A.G.: Weighted composition operators on Hardy spaces. J. Math. Anal. Appl. 263, 224-233 (2001)

5. Cowen, C.C., MacCluer, B.D.: Composition Operators on Spaces of Analytic Functions. Studies in Advanced Mathematics. CRC Press, Boca Raton, FL (1995)

6. Čučković, Z., Zhao, R.: Weighted composition operators between different weighted Bergman spaces and different Hardy spaces. Illinois J. Math. 51, 479-498 (2007)

7. Čučković, Z., Zhao, R.: Weighted composition operators on the Bergman space. J. London Math. Soc. 2(70), 499-511 (2004)

8. Goebeler Jr., T.E.: Composition operators acting between Hardy spaces. Int. Equ. Operator Theory 41, 389-395 (2001)

9. Halmos, P.R.: Measure Theory. Springer-Verlag, New York (1974)

10. Hosokawa, T., Ohno, S.: Differences of composition operators on the Bloch spaces. J. Operator Theory 57, 229-242 (2007) 
11. Moorhouse, J.: Compact differences of composition operators. J. Funct. Anal. 219, 70-92 (2005)

12. Moorhouse, J., Toews, C.: Differences of composition operators. Trends in Banach spaces and operator theory (Memphis, TN, 2001), 207-213, Contemp. Math., 321, Amer. Math. Soc., Providence, RI (2003)

13. Peláez, J.A., Rättyä, J.: Weighted Bergman spaces induced by rapidly increasing weights. Mem. Am. Math. Soc. 227, vi+124 (2014)

14. Peláez, J.A., Rättyä, J.: Bergman projection induced by radial weight. preprint (2019). arXiv: 1902.09837

15. Peláez, J.A., Rättyä, J.: Embedding theorems for Bergman spaces via harmonic analysis. Math. Ann. 362, 205-239 (2015)

16. Peláez, J.A., Rättyä, J.: Trace class criteria for Toeplitz and composition operators on small Bergman spaces. Adv. Math. 293, 606-643 (2016)

17. Peláez, J.A.: Small weighted Bergman spaces. Proceedings of the Summer School in Complex and Harmonic Analysis, and Related Topics, 29-98, Publ. Univ. East. Finl. Rep. Stud. For. Nat. Sci., 22, Univ. East. Finl., Fac. Sci. For., Joensuu (2016)

18. Peláez, J.A., Rättyä, J., Sierra, K.: Berezin transform and Toeplitz operators on Bergman spaces induced by regular weights. J. Geom. Anal. 28, 656-687 (2018)

19. Peláez, J.A., Perälä, A., Rättyä, J.: Hankel operators induced by radial Bekollé - Bonami weights on Bergman spaces. Math. Z. (2019). https://doi.org/10.1007/s00209-019-02412-8

20. Saukko, E.: Difference of composition operators between standard weighted Bergman spaces. J. Math. Anal. Appl. 381, 789-798 (2011)

21. Saukko, E.: An application of atomic decomposition in Bergman spaces to the study of differences of composition operators. J. Funct. Anal. 262, 3872-3890 (2012)

22. Shapiro, J.H.: The essential norm of a composition operator. Ann. of Math. 2(125), 375-404 (1987)

23. Shapiro, J.H.: Composition operators and classical function theory. Universitext: Tracts in Mathematics. Springer-Verlag, New York, xvi+223 pp (1993)

24. Shapiro, J.H., Sundberg, C.: Isolation amongst the composition operators. Pacific J. Math. 145, 117$152(1990)$

25. Smith, W.: Composition operators between Bergman and Hardy spaces. Trans. Am. Math. Soc. 348, 2331-2348 (1996)

26. Smith, W., Yang, L.: Composition operators that improve integrability on weighted Bergman spaces. Proc. Am. Math. Soc. 126, 411-420 (1998)

Publisher's Note Springer Nature remains neutral with regard to jurisdictional claims in published maps and institutional affiliations. 